\title{
ESTABELECIMENTO DE ROTAÇÃO ECONÔMICA PARA UMA FLORESTA REGULADA $^{1}$
}

\author{
Márcio Lopes da Silva² e Carlos Antonio Álvares Soares Ribeiro²
}

\begin{abstract}
RESUMO - O objetivo deste trabalho foi determinar as rotações econômicas para um povoamento florestal, considerando-se um único corte e infinitos cortes, e para uma floresta regulada, por meio de modelos matemáticos, bem como compará-las através da análise marginal das condições de otimalidade. Para validar o modelo e verificar a magnitude dos efeitos, foi utilizado um estudo de caso de um projeto florestal com valores reais de produção, custos, receitas e taxa de desconto. Os resultados indicaram que os modelos foram eficientes para determinar a rotação econômica e que a rotação do povoamento para um único corte e infinitos cortes e para a floresta regulada foi, respectivamente, de 6,5; 5,5; e 5 anos. O Valor Esperado do Solo (VES) para a floresta regulada foi inferior ao VES do povoamento, em virtude de se impor ao manejo a condição de regulação.
\end{abstract}

Palavras-chave: Economia florestal, idade ótima de corte e equações diferenciais.

\section{ESTABLISHMENT OF ECONOMIC ROTATION FOR A REGULATED FOREST}

\begin{abstract}
The objective of this work was to determine the economic rotations for a forest stand taking into considereation only one harvest, infinite harvests and a regulated forest using mathematical models, as well as comparing them through the marginal analysis of optimality conditions. To validate the model and to verify the magnitude of the effect a study case of a forest project, with real values of production, costs, revenues and discounting rate, was used. The results indicated that the models were efficient to determine the economic rotation and that the rotation of the stand for one cut, infinite cuts and for the regulated forest were respectively 6.5, 5.5 and 5 years. The Soil Expectation Value (SEV) for the regulated forest was smaller than the SEV of the stand due toimposed regulations on the forest management.
\end{abstract}

Keywords: Forest economics, rotation age and differential equations.

\section{INTRODUÇÃo}

Para uma floresta destinada à produção de madeira, o período de produção é relativamente longo, portanto o tempo representa fator de produção (NAUTIYAL, 1988). O termo rotação florestal serve para designar a idade na qual povoamentos de mesma idade são planejados para serem colhidos, compreendendo a idade que vai do plantio até o corte da floresta (CLUTTER et al., 1983).
Uma das rotações mais comumente adotadas é a de máxima produtividade, que é obtida quando o Incremento Médio Anual (IMA) é máximo e igual ao Incremento Corrente Anual (ICA). Essa rotação tem sido popularmente utilizada pela sua simplicidade de determinação e pela lógica afirmação de que proporciona a máxima produção volumétrica por unidade de tempo (NAUTIYAL, 1988). Outro tipo de rotação muito empregado é a rotação econômica, que é aquela que proporciona o máximo de lucro ou de rentabilidade

\footnotetext{
${ }^{1}$ Recebido em 21.12.2004 e aceito para publicação em 10.11.2005.

${ }^{2}$ Departamento de Engenharia Florestal da Universidade Federal de Viçosa. E-mail: <marlosil@ufv.br>.
} 
da floresta. Atualmente, com as novas técnicas computacionais, esse tipo de rotação tem sido amplamente utilizado pela facilidade de se incluírem fórmulas financeiras aos programas de manejo florestal. A rotação é um dos fatores mais importantes no processo de planejamento florestal e de regulação da floresta, pois toda a estrutura produtiva é feita com base na rotação (LOPES, 1990).

A regulação da floresta consiste em converter uma estrutura de classes de idade existente de tal forma que, no final do período de transição, as classes de idades estejam em uma progressão aritmética, em que a razão é o intervalo entre colheitas, obtendo-se idades variando de um ano até a idade de rotação (RODRIGUES, 1997). Portanto, a regulação da floresta garante um fluxo anual de madeira e também demanda um fluxo constante de insumos e mão-de-obra ao longo dos anos. Isso é crítico para as empresas florestais que possuem demanda anual estável de madeira para o abastecimento de alguma indústria. Por isso, a regulação da floresta é uma estratégia fundamental para a empresa que busca sustentabilidade (DAVIS e JOHNSON, 1987; LEUSCHNER, 1990).

A maioria dos estudos sobre rotação por meio de modelos matemáticos tem estabelecido a rotação econômica ótima, considerando-se a floresta como se fosse um único povoamento ou talhão, sendo uma área de corte contínua e árvores de mesma idade (HALEY, 1966; PEARSE, 1967; SMITH, 1989; LOPES, 1990; SILVA, 1991; RIBEIRO e GRAÇA, 1996).

Vários trabalhos já apontaram que a rotação econômica de um povoamento destinado a um único corte é maior que a rotação quando se consideram infinitos ciclos de corte (NAUTIYAL,1988; LOPES, 1990; RIBEIRO e GRAÇA, 1996).

Quando se trata, porém, de floresta regulada, o objetivo é maximizar o lucro desta e não do talhão ou povoamento individual. Por isso, há de se considerarem todos os talhões ou compartimentos que estão interrelacionados, espacial e temporalmente.

Nautiyal (1988) e Brum Neto (2001) são uns dos poucos autores que apresentaram a rotação econômica de floresta regulada, por meio de equações diferenciais. O primeiro apenas apresenta um modelo e compara com a rotação de um povoamento com um único corte, infinitos cortes e para a floresta regulada. Porém, seu modelo é simples e não contém os principais custos do projeto florestal e também não utiliza valores numéricos, por isso não dá para saber a magnitude dos efeitos ou quanto essas rotações são diferentes. Já o trabalho de Brum Neto (2001) foi relacionado a povoamentos mistos utilizando um desenho espacial com diferentes culturas numa mesma área, mas também não apresenta valores numéricos.

Os estudos mais recentes sobre regulação de florestas têm utilizado técnicas de programação matemática, como programação linear (Modelo I e Modelo II), e meta-heurística (algorítimos genéticos, busca tabu, "simulating annealing”) (SILVA, 2001; RODRIGUES, 2001). Essas técnicas são complexas na sua modelagem e exigem grande conhecimento de programas computacionais para seu perfeito funcionamento, mas são técnicas bastante promissoras. O fato é que, quando se permite que a rotação seja incluída como uma variável, isso pode levar a uma combinação muito elevada de alternativas de manejo a serem verificadas, o que aumenta a dificuldade na modelagem (RODRIGUES, 2001). Por isso, muitos trabalhos utilizando tais técnicas têm considerado a rotação fixa, ou seja, aquela determinada para o povoamento. Porém, o correto seria utilizar a rotação ótima para a floresta regulada.

Este trabalho teve como objetivo determinar a rotação econômica ótima para a floresta regulada e confrontála com os modelos tradicionais de rotação de um povoamento, considerando-se um único corte e infinitos cortes. Especificamente, pretendeu-se:

- Desenvolver modelos matemáticos para povoamento com um corte, infinitos cortes e para a floresta regulada.

- Comparar os modelos por meio de um estudo de caso utilizando dados de um projeto florestal.

\section{MATERIAL E MÉTODOS}

\subsection{Modelo a-Determinação da rotação econômica para povoamento considerando um único corte}

Considere o seguinte fluxo de caixa de um projeto de reflorestamento:

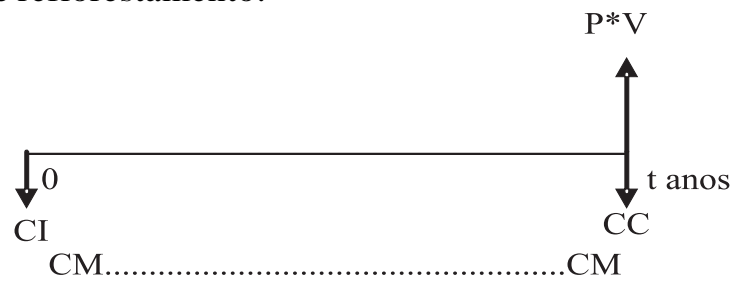


em que:

$\mathrm{CI}=$ custo de implantação da floresta (US\$/ha);

$\mathrm{CM}=$ custo de manutenção anual (US\$/ha);

$\mathrm{CC}=$ custo de colheita (US $\left.\$ / \mathrm{m}^{3}\right)$;

$\mathrm{P}=$ preço da madeira empilhada (US $\left.\$ / \mathrm{m}^{3}\right)$;

$\mathrm{V}=$ produção volumétrica $\left(\mathrm{m}^{3} / \mathrm{ha}\right)$; e

$\mathrm{t}$ = rotação ótima (em anos).

Utilizando-se capitalização contínua, o Valor Presente Líquido que representa o lucro que o reflorestamento proporciona corrigido para o momento zero pode ser dado pela seguinte expressão:

$$
V P L=\frac{P V-C C}{e^{\rho t}}-C I-\frac{C M\left(1-e^{-\rho t}\right)}{e^{\rho}-1}
$$

em que:

VPL = Valor Presente Líquido (US\$/ha);

$\mathrm{r}=$ taxa de desconto anual; e

$\mathrm{e}=$ base dos logarítimos neperianos.

A rotação econômica ou a idade ideal de corte desse povoamento fundamenta-se na maximização do VPL, portanto:

$\frac{\partial V P L}{\partial t}=0 \Rightarrow \frac{\left(P \frac{\partial V}{\partial t}-\frac{\partial C C}{\partial t}\right) e^{\rho t}-(P V-C C) \rho e^{\rho t}}{e^{2 \rho t}}-\frac{\rho C M e^{-\rho t}}{e^{\rho}-1} \Rightarrow$

$\frac{e^{\rho t}}{e^{2 \rho t}}\left[P \frac{\partial V}{\partial t}-\frac{\partial C C}{\partial t}-\rho(P V-C C)\right]=\frac{\rho C M e^{-\rho t}}{e^{\rho}-1} \Rightarrow$

$P \frac{\partial V}{\partial t}-\frac{\partial C C}{\partial t}-(P V-C C) p=\frac{\rho C M}{e^{\rho}-1} \Rightarrow$

$P \frac{\partial V}{\partial t}=\rho(P V-C C)+\frac{\rho C M}{e^{\rho}-1}+\frac{\partial C C}{\partial t}$

Cabe ressaltar que, para a maximização da função, é necessário atender às condições de $2^{\text {a }}$ ordem, ou seja:

$$
\frac{\partial^{2} V P L}{\partial t^{2}}<0
$$

Pela conformação da curva de produção floresta e da curva do VPL no intervalo de tempo que está analisando (Figura 3), percebe-se claramente que se trata de um ponto de máximo. Portanto, essa $2^{\mathrm{a}}$ condição também é atendida.

\subsection{Modelo b - Determinação da rotação econômica para povoamento considerando infinitos cortes}

Nas empresas florestais ocorre que, após o corte, repete-se todo o processo de implantação, manutenção e colheita novamente. Considerando que a terra será utilizada para a atividade florestal indefinidamente, pode-se dizer que o processo tende para infinitos ciclos de produção, conforme o fluxo de caixa a seguir:

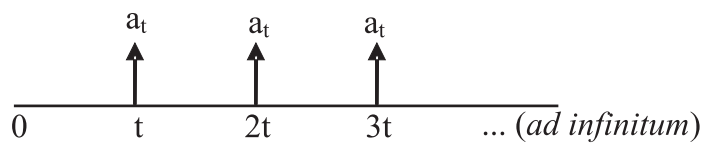

em que:

$\mathrm{a}_{\mathrm{t}}=$ receita líquida da floresta que se repete no final de cada ciclo de $t$ anos. fórmula:

O termo $a_{t}$ pode ser representado pela seguinte

$$
a_{t}=P V-C C-C I e^{\rho t}-\frac{C M\left(e^{\rho t}-1\right)}{e^{\rho}-1}
$$

Como o termo $\mathrm{a}_{\mathrm{t}}$ se repete infinitamente, pode se dizer que o fluxo de caixa anteriormente se refere a uma série infinita postecipada, e o valor presente dessa série representa o Valor Esperado do Solo (VES):

$$
V E S=\frac{a_{t}}{e^{\rho t}-1}
$$

Multiplicando e dividindo a expressão (4) por $\mathrm{e}^{-\mathrm{pt}}$, tem se:

$$
\begin{aligned}
& V E S=\frac{a_{t}}{e^{\rho t}-1} \cdot \frac{e^{-\rho t}}{e^{-\rho t}}=\frac{a_{t} \cdot e^{-\rho t}}{1-e^{-\rho t}} \Rightarrow \\
& V E S=\frac{a_{0}}{1-e^{-\rho t}}
\end{aligned}
$$

em que $\mathrm{a}_{0}$ representa o valor presente líquido de um ciclo da floresta:

$$
\begin{aligned}
& a_{0}=a_{t} \cdot e^{-\rho t} \\
& \text { ou } a_{0}=\frac{P V-C C}{e^{\rho t}}-C I-\frac{C M\left(1-e^{-\rho t}\right)}{e^{\rho}-1}
\end{aligned}
$$

Para obter a rotação ótima, ou seja, aquela que maximiza o VES, basta derivar a sua função em relação a t:

R. Árvore, Viçosa-MG, v.30, n.1, p.65-73, 2006 


$$
\begin{gathered}
\frac{\partial V E S}{\partial t}=0 \Rightarrow \frac{\partial\left(\frac{a_{0}}{1-e^{-\rho t}}\right)}{\partial t}=0 \Rightarrow \\
\left(\frac{1}{1-e^{-\rho t}}\right) \frac{\partial a_{0}}{\partial t}+a_{0} \cdot \frac{\partial\left(\frac{1}{1-e^{-\rho t}}\right)}{\partial t}=0
\end{gathered}
$$

Derivando o $2^{\circ}$ termo da equação (8):

$$
\frac{\partial\left(\frac{1}{1-e^{-\rho t}}\right)}{\partial t}=-\frac{\rho \cdot e^{-\rho t}}{\left(1-e^{-\rho t}\right)^{2}}
$$

Substituindo na equação (8):

$$
\begin{aligned}
& \left(\frac{1}{1-e^{-\rho t}}\right) \frac{\partial a_{0}}{\partial t}=a_{0} \cdot \frac{\rho \cdot e^{-\rho t}}{\left(1-e^{-\rho t}\right)^{2}} \Rightarrow \\
& \frac{\partial a_{0}}{\partial t}=\rho \cdot V E S \cdot e^{-\rho t}
\end{aligned}
$$

Derivando a equação (7) e igualando à equação (10), tem-se:

$$
\frac{\partial a_{0}}{\partial t}=\frac{\left(P \frac{\partial V}{\partial t}-\frac{\partial C C}{\partial t}\right) e^{\rho t}-(P V-C C) \rho e^{\rho t}}{e^{2 \rho t}}-\frac{\rho C M e^{-\rho t}}{e^{\rho}-1}=\rho \cdot V E S \cdot e^{-\rho t}
$$

Colocando o termo $\frac{e^{\rho t}}{e^{2 \rho t}}$ em evidência:

$$
\frac{e^{\rho t}}{e^{2 \rho t}}\left[P \frac{\partial V}{\partial t}-\frac{\partial C C}{\partial t}-(P V-C C) \rho\right]-\frac{\rho C M e^{-\rho t}}{e^{\rho}-1}=\rho \cdot V E S \cdot e^{-\rho t}
$$

Multiplicando toda a expressão por $\mathrm{e}^{\mathrm{pt}}$, tem-se:

$$
P \frac{\partial V}{\partial t}-\frac{\partial C C}{\partial t}-(P V-C C) \rho-\frac{\rho C M}{e^{\rho}-1}=\rho V E S
$$

Logo, pode-se escrever que:

$$
P \frac{\partial V}{\partial t}=\rho(P V-C C)+\frac{\rho C M}{e^{\rho}-1}+\frac{\partial C C}{\partial t}+\rho V E S
$$

Admitindo-se que as condições de $2^{\mathrm{a}}$ ordem estejam satisfeitas:

$$
\frac{\partial^{2} V E S}{\partial t^{2}}<0
$$

R. Árvore, Viçosa-MG, v.30, n.1, p.65-73, 2006
2.3. Modelo c - Determinação da rotação econômica para uma floresta regulada

\begin{tabular}{|c|c|c|c|c|c|c|}
\hline & \multicolumn{6}{|c|}{ Talhões } \\
\hline \multirow{6}{*}{$\begin{array}{l}\text { 여 } \\
\frac{0}{0} \\
0 \\
0 \\
0 \\
0\end{array}$} & & 1 & 2 & 3 & $\ldots$ & $\mathrm{t}$ \\
\hline & 1 & & & & $\ldots$ & \\
\hline & 2 & & & & $\ldots$ & \\
\hline & 3 & & & & $\ldots$ & \\
\hline & $\ldots$ & $\ldots$ & $\ldots$ & $\ldots$ & & $\ldots$ \\
\hline & $t$ & 10 & & & - & \\
\hline
\end{tabular}

Para obter uma floresta regulada, deve-se implantar a cada ano um talhão ou compartimento. Assim, no momento zero se implanta o talhão 1 , no ano seguinte o talhão 2, e assim sucessivamente. Após “ $t$ ” anos teriam sido implantados “t” talhões, e o primeiro estaria no ponto de corte. A partir desse momento, então a floresta estaria regulada, pois a cada ano estaria se implantando e colhendo um talhão, conforme mostrado na Figura 1.

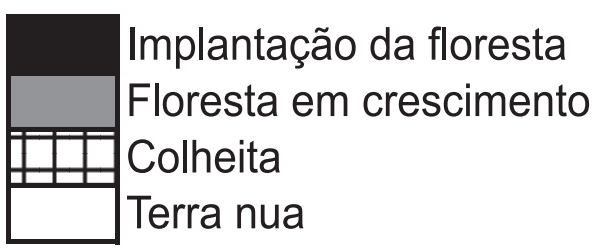

Figura 1 - Seqüência de implantação dos talhões para obtenção de uma floresta regulada.

Figure 1 - Sequence of stand implantation for obtaining a regulated forest.

Portanto, é necessário definir algumas variáveis:

$\mathrm{A}$ = área total da floresta a ser regulada;

$\mathrm{t}$ = rotação ótima, em anos, que é igual ao número de compartimentos ou talhões;

$\mathrm{A} / \mathrm{t}=$ área de plantio e corte anual; e

CI, CM, CC, P, V, $\rho$, conforme definidos anteriormente.

O valor presente líquido $\left(\mathrm{a}_{0}\right)$ para o primeiro ciclo do primeiro talhão pode ser dado pela equação:

$$
a_{0}=\frac{P V-C C}{e^{\rho t}}-C I-\frac{C M\left(1-e^{-\rho t}\right)}{e^{\rho}-1}
$$


Considerando-se que dentro desse talhão 1 o ciclo se repete a cada $t$ anos, o seu fluxo de caixa pode ser ilustrado da seguinte forma:

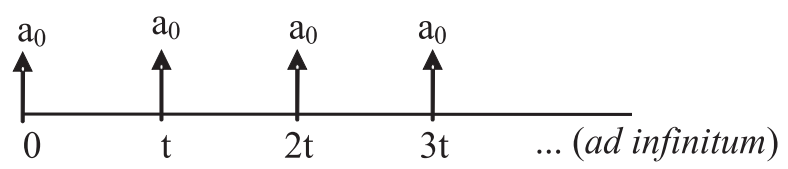

Dessa forma, pode-se estabelecer o VES para o talhão 1:

$$
V E S_{1}=\frac{a_{0} \cdot e^{\rho t}}{e^{\rho t}-1}
$$

Multiplicando e dividindo a expressão (13) por $\mathrm{e}^{-\mathrm{pt}}$, tem se:

$$
V E S_{1}=\frac{a_{0}}{1-e^{-\rho t}}
$$

O segundo talhão 2 é plantado um ano após o talhão 1, sendo o seu fluxo de caixa idêntico ao primeiro, porém defasado de um ano:

$$
V E S_{2}=\frac{V E S_{1}}{e^{\rho}}
$$

De forma análoga, o VES do i-ésimo talhão também será dado por:

$$
V E S_{i}=\frac{V E S_{1}}{e^{\rho(i-1)}}
$$

O VES para a floresta regulada, como um todo, deverá ser a soma dos VES de cada um dos “t” talhões:

$$
V E S_{F R E G}=\sum_{i=1}^{t} V E S_{1} \cdot e^{-\rho(i-1)}
$$

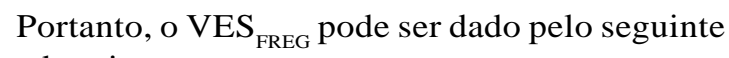
fluxo de caixa:

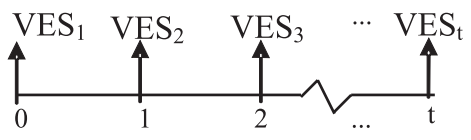

e ser expresso pela seguinte fórmula:

$$
V E S_{F R E G}=\frac{V E S_{1}\left[1-e^{-\rho t}\right]}{e^{\rho}-1}
$$

Substituindo a expressão (14) em (15), tem-se:

$$
\begin{aligned}
V E S_{\text {FREG }} & =\frac{a_{0}\left[1-e^{-\rho t}\right]}{\left(e^{\rho}-1\right)\left[1-e^{-\rho t}\right]} \Rightarrow \\
V E S_{\text {FREG }} & =\frac{a_{0}}{e^{\rho}-1}
\end{aligned}
$$

A equação (16) refere-se ao valor do VES para a floresta total ou a soma dos " $t$ ” talhões. Para se obter o VES por ha, tem que dividir pelo número de talhões que compõem a floresta regulada; logo:

$$
V E S_{F R E G}=\frac{a_{0}}{\left(e^{\rho}-1\right)}
$$

De acordo com a equação (17), a partir do ano t em cada ano será cortado um compartimento de tamanho $\mathrm{A} / \mathrm{t}$, sendo o seu lucro $\mathrm{a}_{0} / \mathrm{t}$, que se repete infinitamente.

Para obter a rotação ótima, ou seja, aquela que maximiza o VES, basta derivar a sua função em relação a t:

$$
\begin{aligned}
& \frac{\partial V E S_{F R E G}}{\partial t}=0 \Rightarrow \frac{\frac{\partial a_{0}}{\partial t}\left(e^{\rho}-1\right) t-a_{0}\left(e^{\rho}-1\right)}{\left[\left(e^{\rho}-1\right) t\right]^{2}}=0 \Rightarrow \\
& \frac{\left(e^{\rho}-1\right) t\left(\frac{\partial a_{0}}{\partial t}-a_{0} \frac{1}{t}\right)}{\left[\left(e^{\rho}-1\right) t\right]^{2}}=0 \Rightarrow \frac{\partial a_{0}}{\partial t}-a_{0} \frac{1}{t}=0 \Rightarrow \\
& \frac{\partial a_{0}}{\partial t}=\frac{a_{0}}{t}
\end{aligned}
$$

Derivando agora a equação (12) referente ao $a_{0}$, tem-se:

$$
\frac{\partial a_{0}}{\partial t}=\frac{\left(P \frac{\partial V}{\partial t}-\frac{\partial C C}{\partial t}\right) e^{\rho t}-(P V-C C) \rho e^{\rho t}}{e^{2 \rho t}}-\frac{\rho C M e^{-\rho t}}{e^{\rho}-1}
$$

Colocando o termo $\frac{e^{\rho t}}{e^{2 \rho t}}$ em evidência e simplificando, tem-se:

$$
\frac{\partial a_{0}}{\partial t}=\frac{1}{e^{\rho t}}\left[P \frac{\partial V}{\partial t}-\frac{\partial C C}{\partial t}-(P V-C C) \rho\right]-\frac{\rho C M e^{-\rho t}}{e^{\rho}-1}
$$

Substituindo (19) em (18):

$\frac{1}{e^{\rho t}}\left[P \frac{\partial V}{\partial t}-\frac{\partial C C}{\partial t}-(P V-C C) \rho\right]-\frac{\rho C M e^{-\rho t}}{e^{\rho}-1}=\frac{a_{0}}{t}$

$\left[P \frac{\partial V}{\partial t}-\frac{\partial C C}{\partial t}-(P V-C C) \rho\right]=\frac{e^{\rho t} a_{0}}{t}+\frac{\rho C M e^{-\rho t}}{e^{\rho}-1} \cdot e^{\rho t}$

$P \frac{\partial V}{\partial t}=\rho(P V-C C)+\frac{\rho C M}{e^{\rho}-1}+\frac{\partial C C}{\partial t}+\frac{a_{t}}{t}$

sendo $a_{t}=a_{0} \cdot e^{\rho t}$ 


\subsection{Estudo de caso}

Para facilitar o entendimento dos modelos, verificar a sua aplicação e também ilustrar as diferenças entre as rotações econômicas do povoamento de um corte, infinitos cortes e da floresta regulada, foi desenvolvido um estudo de caso de um projeto de reflorestamento com eucalipto.

O exemplo utilizado para ilustrar este trabalho teve como base um modelo de crescimento e produção (modelo de Clutter), ajustado para uma situação real por Soares (1999), com o híbrido Eucalyptus grandis x Eucalyptus urophylla, produzido a partir de estacas, considerando-se a idade-índice de 60 meses. Esse modelo é apresentado no Quadro 1.

Utilizou-se neste estudo um índice de local de 24 e área basal inicial de 13 m²/ha.

Os demais dados do projeto são apresentados no Quadro 2.

Quadro 1 - Modelos de crescimento e produção (modelo de Clutter) genérico e ajustado por Soares (1999), para uma situação real

Table 1 - Growth and production model (Clutter's Model) adjusted by Soares (1999), for a real situation

\begin{tabular}{|c|}
\hline Modelo Genérico \\
\hline 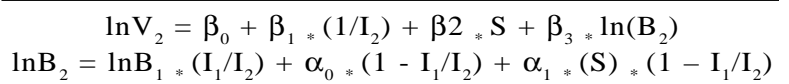 \\
\hline
\end{tabular}

$\mathrm{LnV}_{2}=1,81969-25,712511 / \mathrm{I}_{2}+0,02073 * \mathrm{~S}+1,13070_{*} \ln \mathrm{B}_{2}$ $\mathrm{LnB}_{2}=\operatorname{lnB}_{1^{*}}\left(\mathrm{I}_{1} / \mathrm{I}_{2}\right)+2,96228_{*}\left(1-\mathrm{I}_{1} / \mathrm{I}_{2}\right)+0,01533_{*} \mathrm{~S}_{*}\left(1-\mathrm{I}_{1} / \mathrm{I}_{2}\right)$

em que $\mathrm{ln}=$ logaritmo neperiano; $\mathrm{S}$ = índice de local, em $\mathrm{m} ; \mathrm{B}_{1}$ = área basal corrente, em $\mathrm{m}^{2} / \mathrm{ha} ; \mathrm{V}_{2}=$ volume futuro, em $\mathrm{em}^{3} / \mathrm{ha}$; $\mathrm{B}_{2}$ = área basal futura, em $\mathrm{m}^{2} / \mathrm{ha} ; \mathrm{I}_{1}=$ idade atual, em meses; $\mathrm{I}_{2}$ = idade futura, em meses; e $b_{0} a_{3} b_{3}$ e $a_{0}$ e $a_{1}=$ parâmetros do modelo na forma estrutural.

\section{RESULTADOS E DISCUSSÃO}

\subsection{Modelos matemáticos - equações diferenciais}

Os modelos indicaram que as rotações econômicas nas três formas de manejo são diferentes (Quadro 3), confirmando os mesmos resultados apontados por Nautiyal (1998).

Considerandoas equações(2),(11)e(20), o lado esquerdo destas representa o valor do produto marginal no tempo, ou seja, o ganho obtido por se adiar o corte por mais um ano(ou período). Olado esquerdo representa o custo marginal dessa ação, ou seja, o custo adicional que a empresa tem em postergar o corte por mais um ano.

Considerando-se um único corte, equação (2), o primeiro termo do lado esquerdo, $\rho(P V-C C)$, representa os juros sobre o valor da floresta já colhida, ou o custo de oportunidade da floresta como capital, se a madeira fosse vendida e o dinheiro colocado a juros num banco. O segundo termo, $\frac{\rho C M}{e^{\rho}-1}$, representa o custo de manutenção que se tem por postergar o corte mais um ano. O terceiro termo, $\frac{\partial C C}{\partial t}$, representa o acréscimo do custo de colheita em virtude do crescimento da floresta por mais um ano.

Considerando infinitos cortes, equação (11), o lado esquerdo da equação possui um termo a mais que a equação (2), $\rho V E S$, que é o custo de oportunidade da terra, caso a floresta fosse cortada e a terra alugada ou vendida e o dinheiro colocado a juros num banco.

Considerando a floresta regulada, a equação (20), o quarto termo do lado esquerdo, $\frac{a_{t}}{t}$, representa a perda do lucro proporcionado por um compartimento ou talhão por se ter adiado o corte por mais um ano, portanto representa um custo a mais.

Quadro 2 - Dados de custos e receitas do projeto de reflorestamento

Table 2 - Data of costs and revenues of the reforestation project

\begin{tabular}{lccc}
\hline Itens de Projeto & Sigla & Ano de Ocorrência & Valor (US\$) \\
\hline Custo de implantação & $\mathrm{CI}$ & 0 & 650,00 US\$/ha \\
Custo de manutenção anual* & $\mathrm{CM}$ & Anualmente & 90,00 US\$/ha \\
Custo de colheita & $\mathrm{CC}$ & $\mathrm{t}$ & $3,00 \mathrm{US}^{*} / \mathrm{m}^{3}$ \\
Volume de madeira & $\mathrm{V}$ & $\mathrm{t}$ & $\mathrm{Equação} \mathrm{do} \mathrm{Quadro} 1$ \\
Taxa de juros & $\mathrm{r}$ & - & $10 \%$ a.a. \\
Preço da madeira & $\mathrm{P}$ & - & $18,00 \mathrm{US}^{3} / \mathrm{m}^{3}$ \\
\hline
\end{tabular}

*Inclui os custos de administração, conservação de estradas e aceiros e combate a formigas (exclui o custo da terra). 
Observa-se que em nenhum dos três casos apareceu o custo de implantação (CI), ou seja, por ele aparecer uma única vez, no momento inicial, ele não afeta as rotações analisadas. Esse fato foi também observado por Lopes (1998) e Silva (1991).

\subsection{Estudo de caso}

\subsubsection{Modelos de crescimento e produção}

A Figura 2 ilustra o cruzamento das curvas de incremento médio anual e incremento corrente anual, indicando que a rotação técnica ocorre aos cinco anos.

No Quadro 4, mostram-se os valores do lucro descontado, o valor do produto marginal e o custo marginal em função da idade, nas três modalidades de manejo.

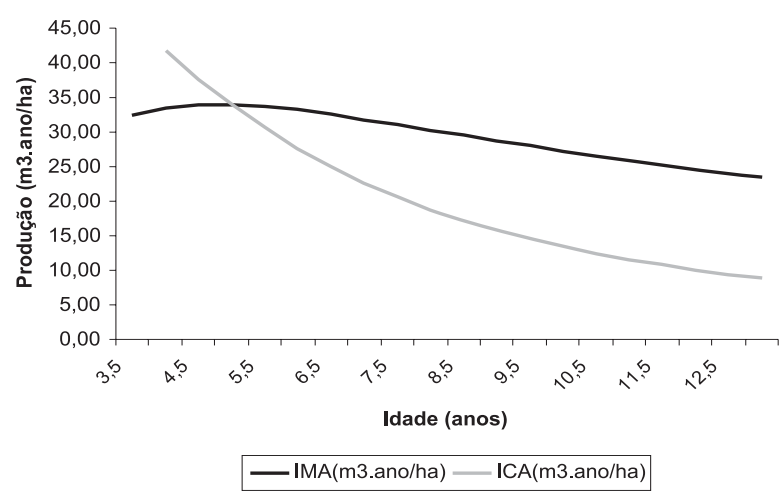

Figura 2 - Curvas do incremento médio anual e incremento corrente anual, indicando a idade técnica de corte.

Figure 2 - Annual average increment and annual current increment curves, indicating the technique rotation.

Quadro 3 - Condições de otimalidade nas diferentes formas de manejo

Table 3 - Optimality conditions for the different forms of management

\begin{tabular}{lcc}
\hline Tipo de Manejo & Equação & Condições de Otimalidade \\
\hline Povoamento - único corte & (2) & $P \frac{\partial V}{\partial t}=\rho(P V-C C)+\frac{\rho C M}{e^{\rho}-1}+\frac{\partial C C}{\partial t}$ \\
Povoamento - infinitos cortes & $(11)$ & $P \frac{\partial V}{\partial t}=\rho(P V-C C)+\frac{\rho C M}{e^{\rho}-1}+\frac{\partial C C}{\partial t}+\rho V E S$ \\
Floresta regulada & $(20)$ & $P \frac{\partial V}{\partial t}=\rho(P V-C C)+\frac{\rho C M}{e^{\rho}-1}+\frac{\partial C C}{\partial t}+\frac{a_{t}}{t}$ \\
\hline
\end{tabular}

Quadro 4 - Produção, lucro atualizado, valor do produto marginal e custo marginal, do povoamento em um único corte (modelo a) e infinitos cortes (modelo b) e para a floresta regulada (modelo c) ao longo dos anos

Table 4 - Production, discounted profit, marginal product value and marginal cost, for the stand in only one cut (model $a$ ), infinite cuts (model $b$ ) and for the regulated forest (model c) along the years

\begin{tabular}{|c|c|c|c|c|c|c|c|c|}
\hline \multirow[t]{2}{*}{ Idade } & \multirow[t]{2}{*}{ Produção } & Modelo a & Modelo b & Modelo c & \multirow[b]{2}{*}{$\mathrm{PdV} / \mathrm{dt}$} & Modelo a & Modelo b & Modelo c \\
\hline & & VPL & VES & VES & & \multicolumn{3}{|c|}{ Custo Marginal do Fator } \\
\hline (anos) & m3/ha & & & & US\$/ha & & & \\
\hline 3,5 & 113,16 & 293,43 & 993,62 & 838,36 & & & & \\
\hline 4,0 & 134,03 & 415,49 & $1.260,27$ & $1.038,72$ & 751,19 & 411,81 & 537,84 & 566,77 \\
\hline 4,5 & 152,88 & 502,12 & $1.385,66$ & $1.115,83$ & 678,78 & 428,03 & 566,59 & 603,02 \\
\hline 5,0 & 169,86 & 558,65 & $1.419,81$ & $1.117,30$ & 611,16 & 442,22 & 584,20 & 626,43 \\
\hline 5,5 & 185,14 & 590,23 & $1.395,18$ & $1.073,15$ & 550,19 & 454,98 & 594,50 & 640,99 \\
\hline 6,0 & 198,92 & 601,46 & $1.333,06$ & $1.002,43$ & 496,12 & 466,65 & 599,95 & 649,30 \\
\hline 6,5 & 211,38 & 596,25 & $1.247,51$ & 917,31 & 448,53 & 477,40 & 602,15 & 653,12 \\
\hline 7,0 & 222,68 & 577,90 & $1.147,96$ & 825,57 & 406,75 & 487,39 & 602,18 & 653,64 \\
\hline 7,5 & 232,96 & 549,11 & $1.040,71$ & 732,15 & 370,06 & 496,69 & 600,76 & 651,69 \\
\hline 8,0 & 242,34 & 512,14 & 930,02 & 640,17 & 337,79 & 505,39 & 598,39 & 647,86 \\
\hline 8,5 & 250,94 & 468,81 & 818,76 & 551,54 & 309,33 & 513,53 & 595,41 & 642,57 \\
\hline 9,0 & 258,83 & 420,65 & 708,84 & 467,39 & 284,16 & 521,18 & 592,06 & 636,14 \\
\hline 9,5 & 266,10 & 368,89 & 601,52 & 388,30 & 261,81 & 528,36 & 588,51 & 628,77 \\
\hline 10,0 & 272,82 & 314,54 & 497,60 & 314,54 & 241,92 & 535,13 & 584,89 & 620,63 \\
\hline 10,5 & 279,05 & 258,45 & 397,57 & 246,14 & 224,14 & 541,50 & 581,26 & 611,84 \\
\hline 11,0 & 284,83 & 201,28 & 301,71 & 182,98 & 208,20 & 547,52 & 577,69 & 602,49 \\
\hline 11,5 & 290,22 & 143,60 & 210,14 & 124,87 & 193,86 & 553,21 & 574,22 & 592,64 \\
\hline 12,0 & 295,24 & 85,87 & 122,88 & 71,56 & 180,92 & 558,59 & 570,88 & 582,35 \\
\hline 12,5 & 299,94 & 28,44 & 39,87 & 22,76 & 169,21 & 563,69 & 567,68 & 571,63 \\
\hline 13,0 & 304,35 & $(28,37)$ & $(39,00)$ & $(21,82)$ & 158,59 & 568,53 & 564,63 & 560,52 \\
\hline
\end{tabular}


A análise marginal indica que a rotação econômica ótima é de 6,5; 5,5; e 5 anos para os modelos a, b e c, respectivamente. Pois é próximo dessa idade que VPL, VES

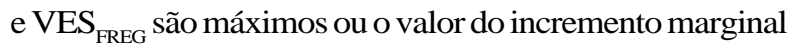
se iguala ao custo marginal do fator. Tal fato pode ser observado no Quadro 4 e também nas Figuras 3 e 4.

A Figura 3 ilustra as funções de lucro nos diferentes regimes de manejo. Quando se trata do manejo do povoamento com um único corte, o lucro é bem menor (curva do VPL), principalmente se as taxas de desconto são elevadas. O lucro mais alto se dá ao manejar o povoamento, visando infinitos cortes (curva do VES), mas essa situação somente permite a produção de madeira ao final de cada ciclo de t anos, ou seja, não permite um fluxo contínuo de madeira ao longo dos anos. Para conseguir obter um fluxo anual de colheitas, é necessário regular a floresta. Nesse caso, o lucro é um pouco menor que no modelo b. Pode-se dizer que essa diferença entre os lucros dos modelos b e c é o custo que se tem ao impor uma restrição de regulação na floresta, ou seja, é o custo da regulação.

A Figura 4 ilustra, com maior clareza, a diferença entre as idades econômicas de corte entre os modelos estudados. Confirmando, assim, que a floresta regulada apresenta uma rotação menor que as demais, pois sua curva de custo marginal é mais alta.

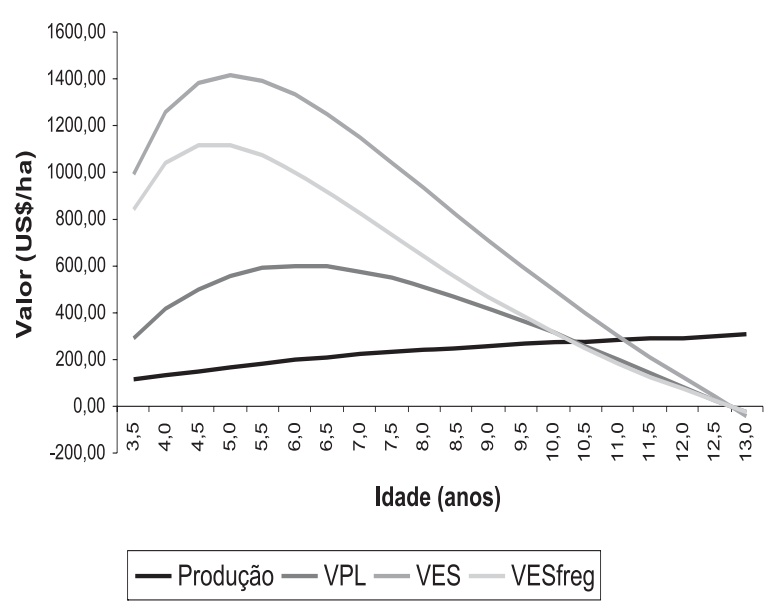

Figura 3 - Curvas da produção, VPL, VES e VES ${ }_{\text {FREG }}$ em função da idade da floresta.

Figure 3-Production, VPL, VES and VES ${ }_{F R E G}$ curves in function of forest age.

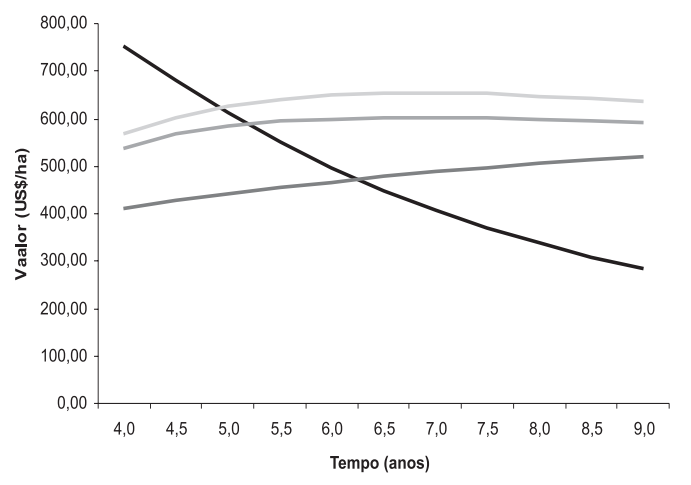

$\begin{array}{ll}-P d V / d t & -\rho P V-\rho C C+\rho C M / e \rho-1+d C C / d t \\ -\rho P V-\rho C C+\rho C M / e \rho-1+d C C / d t+\rho V E S & -\rho P V-\rho C C+\rho C M / e \rho-1+d C C / d t+a t / t\end{array}$

Figura 4-Curvas do valor do incremento marginal e dos custos marginais do fator nos modelos a, b e c em função da idade, indicando a rotação ótima.

Figure 4-Marginal increment value and the marginal costs of the factor curves for the models $a, b$ and $c$ in function of the age, indicating the optimum rotation.

\section{CONCLUSÕES}

As principais conclusões deste trabalho foram:

- Os modelos foram consistentes e eficientes para obtenção da rotação econômica.

- As rotações para cada modalidade de manejo são diferentes.

- A floresta regulada apresenta a rotação inferior, pois mostra um componente a mais no seu custo marginal do fator.

- O lucro descontado da floresta regulada $\left(\operatorname{VES}_{\mathrm{FREG}}\right)$ é menor que o VES do povoamento manejado em infinitos cortes. Essa diferença de valor pode ser considerada o custo da regulação.

- Quando o objetivo é a maximização do lucro de uma floresta regulada, deve-se atentar para o fato de que os talhões estão dependentes, com relação à sua distribuição espacial e temporal.

\section{REFERÊNCIAS BIBLIOGRÁFICAS}

BRUM NETO, M. S. Análise econômica do manejo sustentável de reflorestamentos mistos. 2001. 30 f. Dissertação (Mestrado em Ciência Florestal) - Universidade Federal de Viçosa, Viçosa, 2001. 
CLUTTER, J. L. et al. Timber management: a quantitative approach. Flórida: Krieger Publishing Company, 1983. 333 p.

DAVIS, L. S.; JOHNSON, K. N. Forest management. 3. ed. New York: McGraw-Hill, 1987. $790 \mathrm{p}$.

HALEY, D. The importance of land opportunity cost in the determination of financial rotations. Journal of forestry, n.64, v.5, p. 326-329, 1966.

LEUSCHNER, W. A. Forest regulation, harvest scheduling, and planning techniques. New York: John Wiley \& Sons, 1990. 281 p.

LOPES, H.V.S. Análise econômica dos fatores que afetam a rotação de povoamentos de eucaliptos. 1990. $188 \mathrm{f}$. Dissertação (Mestrado em Ciência Florestal) Universidade Federal de Viçosa, Viçosa, 1990.

NAUTIYAL, J. C. Forest economics: principles and applications. Toronto: Canadian Scholars’ Press, 1988. 581 p.

PEARSE, P.H. The optimum forest rotation. The Forestry Chronicle, n.43, v.2, p.178-195, 1967.

RIBEIRO, C.A.A.S.; GRAÇA, L.R. Manejo por talhadias: estabelecimento das idades ótimas de corte. Revista Árvore, v.20, n.1, p.29-36, 1996.
RODRIGUES, F. L. Regulação de florestas eqüiâneas utilizando programação linear. 1997. 117f. Dissertação (Mestrado em Ciência Florestal) - Universidade Federal de Viçosa, Viçosa, 1997.

RODRIGUES, F. L. Metaheurística e sistema de suporte à decisão no gerenciamento de recursos florestais. 2001. 255f. Dissertação (Doutorado em Ciência Florestal) - Universidade Federal de Viçosa, Viçosa, 2001.

SILVA, A.A.L. Análise econômica da substituição de povoamentos de eucaliptus spp. 1991. 109f. Tese (Mestrado em Ciência Florestal) - Universidade Federal de Viçosa, Viçosa, 1991.

SILVA, G. F. Problemas no uso de programação matemática e simulação em regulação florestal. 2001. 89 f. Tese (Doutorado em Ciência Florestal) - Universidade Federal de Viçosa, Viçosa, 2001.

SMITH, E. B. S. Determinação da rotação econômica para Eucalyptus grandis (W. Hill ex Maiden) destinados à produção de carvão vegetal. 1989. 69 f. Dissertação (Mestrado em Ciência Florestal) Universidade Federal de Viçosa, Viçosa, 1989.

SOARES, C. P. B. Um modelo para o gerenciamento da produção de madeira em plantios comerciais de eucalipto. 1999. $71 \mathrm{f}$. Tese (Doutorado em Ciência Florestal) - Universidade Federal de Viçosa, Viçosa, 1999. 